\title{
Genome-wide association study of powdery mildew resistance in collection of common wheat varieties (T. aestivum L.)
}

\author{
Leonova I.N. \\ Institute of Cytology and Genetics, SB RAS, Novosibirsk, Russia \\ e-mail:leonova@bionet.nsc.ru
}

Powdery mildew, caused by the fungal pathogen Blumeria graminis f. sp. tritici (Bgt), is one of the economically important disease of common wheat $T$. aestivum L. One of the most effective and environmentally important ways of wheat protection against $B g t$ is cultivation of the varieties with genetic resistance. The aim of this work was to study the genetic diversity of collection of common wheat varieties and breeding line on resistance to Bgt. Wheat panel consists of 100 Russian wheat cultivars and 60 breeding lines containing introgressions from Triticeae tribe species. The results of the evaluation of the resistance level of wheat cultivars showed that no more than $10 \%$ of the varieties have low level of susceptibility to the population of Bgt, specific to the Western Siberian region. Among introgression lines, more than $30 \%$ showed a moderate to highly resistant infection types. Association mapping, performed on the basis of SNP genotyping and phytopathological evaluation during three environmental seasons identified ten loci in chromosomes 1AL, 1DS, 2AL, 2BL, 5AS, 5DS, 6AL, 6DL, 7AL, and 7BL. A high impact to the phenotypic manifestation of the trait was established for genetic factors localized in chromosomes $5 \mathrm{AS}, 6 \mathrm{AL}$ and $6 \mathrm{DL}$. In the long arm of chromosome $6 \mathrm{D}$, two loci were mapped which provide effective protection from powdery mildew pathogen. One of them was introduced from the wheatgrass Th. intermedium, another - from tetraploid wheat T. timopheevii. Based on comparative analysis of the chromosomal localization of the known Pm resistance genes and loci mapped in this work, an assumption was made that the QTLs on chromosomes 1DS, 2BL, 5AS, 6DL are new, not previously described resistance loci. The obtained results can be used in breeding programs for selection of target loci and for development of molecular markers specific for Bgt resistance loci.

Acknowledgements: GWAS of wheat cultivars was supported by the Russian Science Foundation (project No. 16-16-00011-P); association mapping of QTLs in introgression lines was supported by Russian Foundation for Basic Research (project No. 18-51600001). 